\title{
MATHEMATICAL
}

PROCEEDINGS

(formerly Proceedings)

of the

Cambridge Philosophical Society

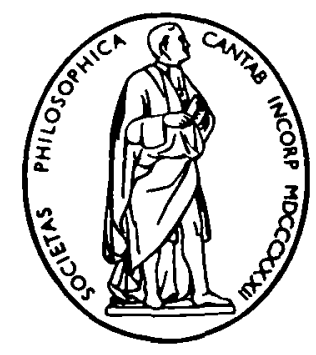


Published by the Press Syndicate of the Cniversity of Cambridge

The Pitt Building, Trumpington Street, Cambridge CB2 1RP 40 West 20th Street, New York, NY 10011-4211, USA

10 Stamford Road, Oakleigh, Melbourne 3166, Australia

(C) Cambridge Philosophical Society 1996

Printed in Great Britain by the University Press, Cambridge 


\section{INDEX FOR VOLUME 119}

Berrick, A. J. \& Tan, G. C. The minimal extension of $P$-localization on groups .

Bray, J. N., Curtis, R. T. \& Hammas, A. M. A. A systematic approach to symmetric presentations I. Involuntary generators .

Brown, B. M., Evans, W. D. \& Ismail, M. E. H. The Askey-Wilson polynomials and $q$-Sturm-Liouville problems

Brown, G., Koumandos, S. \& Wang, K.-Y. Positivity of more Jacobi polynomial sums . 681

Bshouty, D., Hengartner, W. \& Hossian, O. Harmonic typically real mappings . $\quad 673$

Bunce, L. J. \& Hamhalter, J. Extension of Jauch-Piron states on Jordan algebras . . 279

Cariñena, J. F. \& Martínez, E. Geometric characterization of linearisable second-order differential equations

Ciach, L. J., Jajte, R. \& Paszkiewicz, A. On the almost sure approximation of self-adjoint operators in $L_{2}(0,1)$

Cochran, T. D. Non-trivial links and plats with trivial Gassner matrices

Collins, D. J. \& Turner, E. C. All automorphisms of free groups with maximal rank fixed subgroups

Cromwell, P. R. \& Nutt, I. J. Embedding knots and links in an open book II: Bounds on arc index

Curtis, R. T., Hammas, A. M. A. \& Bray, J. N. A systematic approach to symmetric presentations I. Involuntary generators .

Dimov, G. D. \& Tironi, G. Compactifications and A-compactifications of frames. Proximal frames

Doty, S. \& Walker, G. Truncated symmetric powers and modular representations $\mathrm{GL}_{n}$

Dyakonov, K. M. On the zeros and Fourier transforms of entire functions in the PaleyWiener space.

Evans, W. D., Ismail, M. E. H. \& Brown, B. M. The Askey-Wilson polynomials and $q$-Sturm-Liouville problems

Falconer, K. J. \& Howroyd, J. D. Projection theorems for box and packing dimensions . 287

Falconer, K. J. \& Mattila, P. The packing dimension of projections and sections of measures 695

Farhadi, H.-R. Isomorphisms between the second duals of group algebras of locally compact groups

Ferleger, S. V. \& Sukochev, F. A. On the contractibility to a point of the linear groups of non-commutative $L^{p}$-spaces . . . . . . . . . . . . . . . 545

Greenberg, P. Marked homeomorphisms and the realization problem . . . . . . $\quad .575$

Hamhalter, J. \& Bunce, L. J. Extension of Jauch-Piron states on Jordan algebras . . 279

Hammas, A. M. A., Bray, J. N. \& Curtis, R. T. A systematic approach to symmetric presentations I. Involuntary generators. . . . . . . . . . . . . 23

Hatcher, A. \& Oertel, U. Full laminations in 3-manifolds . . . . . . . . . . . . $\quad$. 73

Hengartner, W., Bshouty, D. \& Hossian, O. Harmonic typically real mappings $\quad$. $\quad$. 673

Hitczenko, P. \& Montgomery-Smith, S. J. Tangent sequences in Orlicz and rearrangement invariant spaces

Hossian, O., Hengartner, W. \& Bshouty, D. Harmonic typically real mappings $\quad$. $\quad .673$

Howroyd, J. D. On Hausdorff and packing dimension of product spaces . . . . $\quad 715$

Howroyd, J. D. \& Falconer, K. J. Projection theorems for box and packing dimensions . 287

Huxley, M. N. \& Trifonov, O. The square-full numbers in an interval . . . . . 201 
Ismail, M. E. H., Brown, B. M. \& Evans, W. D. The Askey-Wilson polynomials and $q$-Sturm-Liouville problems

Jajte, R., Paszkiewicz, A. \& Ciach, L. J. On the almost sure approximation of self-adjoint operators in $L_{2}(0,1)$

Jakubec, S. Congruence of Ankeny-Artin-Chowla type for cyclic fields of prime degree I . 17

Jiang, J. F. Sublinear discrete-time order-preserving dynamical systems . . . . . . 561

Joyal, A., Street, R. \& Verity, D. Traced monoidal categories . . . . . . . . . . . 447

Kaniuth, E. Minimal primal ideal spaces and norms of inner derivations of tensor products of $C^{*}$-algebras

Katsoulis, E. G. Reflexivity for a class of subspace lattices.

Kirby, D. \& Rees, D. Multiplicities in graded rings II : integral equivalence and the Buchsbaum-Rim multiplicity

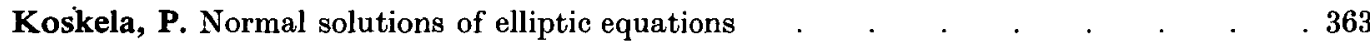

Koumandos, S., Wang, K.-Y. \& Brown, G. Positivity of more Jacobi polynomial sums . 681

Kwasik, S. \& Schultz, R. Fundamental groups of 4-manifolds with circle actions $\quad$. $\quad .645$

Lapidus, M. L. \& Pomerance, C. Counterexamples to the modified Weyl-Berry conjecture on fractal drums

Le Merdy, C. Representation of a quotient of a subalgebra of $B(X)$. . . . . . . . . . 83

Lee, C.-N. Farrell cohomology and centralizers of elementary Abelian p-subgroups . . 403

Luft, E. On 2-dimensional aspherical complexes and a problem of J. H. C. Whitehead $\quad 493$

Majid, S. \& Markl, M. Glueing operation for R-matrices, quantum groups and link-

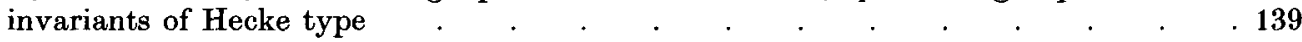

Marar, W. L. \& Tari, F. On the geometry of simple germs of co-rank 1 maps from $\mathbb{B}^{3}$ to $\mathbb{R}^{3} 469$

Markl, M. \& Majid, S. Glueing operation for R-matrices, quantum groups and link-

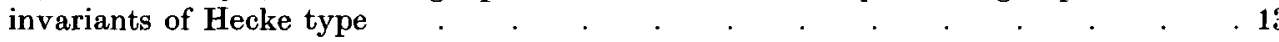

Martínez, E. \& Cariñena, J. F. Geometric characterization of linearisable second-order

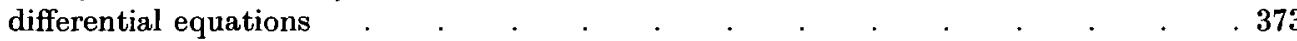

Martino, J. \& Priddy, S. Unstable homotypy classification of $B G_{\hat{p}} \quad$. $\quad . \quad$. $\quad . \quad$. $\quad 119$

Mattila, P. \& Falconer, K. J. The packing dimension of projections and sections of measures 695

Montgomery-Smith, S. J. \& Hitczenko, P. Tangent sequences in Orlicz and rearrangement invariant spaces

Morimoto, K., Sakuma, M. \& Yokota, Y. Examples of tunnel number one knots which have the property ${ }^{\prime} 1+1=3{ }^{\prime} . \quad . \quad . \quad . \quad . \quad . \quad . \quad . \quad . \quad . \quad . \quad .11$

$\mathbf{N g}$, C.-K. Discrete coactions on Hilbert C*-modules . . . . . . . . . . . . 103

Nutt, I. J. \& Cromwell, P. R. Embedding knots and links in an open book II: Bounds on arc index

Oertel, U. \& Hatcher, A. Full laminations in 3-manifolds . . . . . . . . . . . . . 73

Ogi, H. On ground states for CCR algebras and Bogoliubov automorphism groups . . 419

Paszkiewicz, A., Ciach, L. J. \& Jajte, R. On the almost sure approximation of self-adjoint operators in $L_{2}(0,1)$

Peller, V. V. \& Young, N. J. Superoptimal approximation by meromorphic functions

Pomerance, C. \& Lapidus, M. L. Counterexamples to the modified Weyl-Berry conjecture on fractal drums

Powell, G. M. L. Attaching cells to finite complexes, with an application to elliptic spaces 483

Priddy, S. \& Martino, J. Unstable homotopy classification of $B G_{p} \quad$. $\quad . \quad$. $\quad . \quad . \quad 119$

Rees, D. \& Kirby, D. Multiplicities in graded rings II: integral equivalence and the Buchsbaum-Rim multiplicity.

Rein, G. Cosmological solutions of the Vlasov-Einstein system with spherical, plane, and hyperbolic symmetry

Richards, M. J. Some decomposition numbers for Hecke algebras of general linear groups. 383 
Rippon, P. J. Towards a higher-dimensional MacLane class. $\quad . \quad$. $\quad$. $\quad$. $\quad$. $\quad$. 665

Rocha, A. C. Meromorphic extension of the Selberg zeta function for Kleinian groups via thermodynamic formalism . . . . . . . . . . . . . . . . $\quad .179$

Ryba, A. J. E. A natural invariant algebra for the Harada-Norton group . . . . . 597

Sakuma, M., Yokota, Y. \& Morimoto, K. Examples of tunnel number one knots which have the property ' $1+1=3$ '. . . . . . . . . . . . . . 113

Schultz, R. \& Kwasik, S. Fundamental groups of 4-manifolds with circle actions $\quad . \quad$. 645

Stallard, G. M. The Hausdorff dimension of Julia sets of entire functions II . . . $\quad .513$

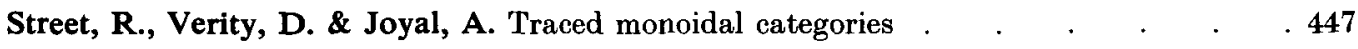

Sukochev, F. A. \& Ferleger, S. V. On the contractibility to a point of the linear groups of non-commutative $L^{p}$-spaces . . . . . . . . . . . . . . . . . 545

Tan, G. C. \& Berrick, A. J. The minimal extension of $P$-localization on groups . $\quad$. $\quad .243$

Tari, F. \& Marar, W. L. On the geometry of simple germs of co-rank 1 maps from $\mathbb{R}^{3}$ to $\mathbb{R}^{3} 469$

Taylor, M. J. The trace form for tame, abelian extensions of number fields $\quad . \quad$. $\quad .209$

Tironi, G. \& Dimov, G. D. Compactifications and A-compactifications of frames. Proximal frames . . . . . . . . . . . . . . . . . 321

Trifonov, O. \& Huxley, M. N. The square-full numbers in an interval . . . . . 201

Truss, J. K. Betweenness relations and cycle-free partial orders . . . . . . . . . . 631

Turner, E. C. \& Collins, D. J. All automorphisms of free groups with maximal rank fixed

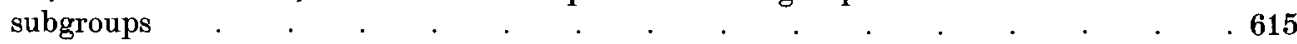

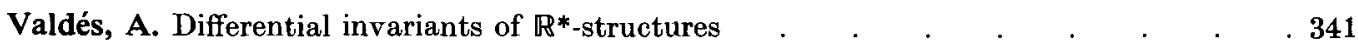

Verity, D., Joyal, A. \& Street, R. Traced monoidal categories _ . . . . . . . . . 447

Walker, G. \& Doty, S. Truncated symmetric powers and modular representations of $\mathrm{GL}_{n} 231$

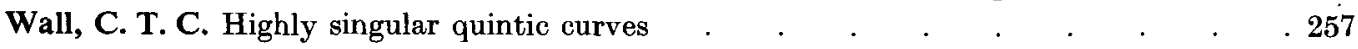

Wang, K.-Y., Brown, G. \& Koumandos, S. Positivity of more Jacobi polynomial sums . 681

Werner, W. Beurling's projection theorem via one-dimensional Brownian motion . . 729

Willerton, S. Vassiliev invariants and the Hopf algebra of chord diagrams. . . . . . 55

Wójcik, J. On a problem in algebraic number theory . _ . . . . . . . . . . . $\quad .191$

Yagita, N. Note on the spectral sequence converging to cohomology of an extra special $p$-group for odd prime $p$. . . . . . . . . . . . . . . . . . . 35

Yokota, Y., Morimoto, K. \& Sakuma, M. Examples of tunnel number one knots which have the property ' $1+1=3$ ' . . . . . . . . . . . . . . . 113

Young, N. J. \& Peller, V. V. Superoptimal approximation by meromorphic functions $\quad .497$ 


\section{The attention of authors is particularly directed to the following requests.}

1. Papers should be typed, double-spaced, on one side of white paper (of which A4, 210 by $297 \mathrm{~mm}$, is a suitable size). The pages must be numbered. Margins of $30 \mathrm{~mm}$ should be left at the side, top and bottom of each page. Two clear copies should be sent.

A cover page should give the title, the author's name and institution, with the address to which mail should be sent.

The title, while brief, must be informative (e.g. A new proof of the prime-number theorem, whereas Some applications of a theorem of $G$. H. Hardy would be useless).

The first paragraph or two should form a summary of the main theme of the paper, providing an abstract intelligible to mathematicians.

For a typescript to be accepted for publication, it must accord with the standard requirements of publishers, and be presented in a form in which the author's intentions regarding symbols etc. are clear to a printer (who is not a mathematician).

The following notes are intended to help the author in preparing the typescript. New authors may well enlist the help of senior colleagues, both as to the substance of their work and the details of setting it out correctly and attractively.

\section{Notation}

Notation should be chosen carefully so that mathematical operations are expressed with all possible neatness, to lighten the task of the compositor and to reduce the chance of error.

For instance $n_{k}(n$ sub $k)$ is common usage, but avoid if possible using $c \operatorname{sub} n \operatorname{sub} k$. Fractions are generally best expressed by a solidus. Complicated exponentials like

should be shown in this and no other way.

$$
\exp \left\{z^{2} \sin \theta /\left(1+y^{2}\right)\right\}
$$

In the manuscript, italics, small capitals and capitals are specified by single, double and triple underlinings. Bold faced type is shown by wavy underlining; wavy will be printed wavy.

It helps if displayed equations or statements which will be quoted later are numbered in order on the right of their line. They can then be referred to by, for example, 'from (7)'.

The author must enable the printer (if necessary by pencilled notes in the margin) to distinguish between similar symbols such as $0, O, \mathrm{o}, \mathrm{O}, 0 ; x, \mathrm{X}, \mathrm{X} ; \dot{\phi}, \Phi, \varnothing ; 1,1 ; \hat{\mathrm{A}}, \in ; \hat{\mathrm{I}}, k$.

Greek letters can be denoted by Gk in the margin.

If an author wishes to mark the end of the proof of a theorem, the sign I may be used.

Footnotes should be avoided.

\section{Diagrams}

It is extremely helpful if diagrams are drawn in Indian ink on white card, faintly blue or green-lined graph paper, or tracing cloth or paper. Symbols, legends and captions should be given on a transparent overlay. Each text figure must be numbered as Figure 1, Figure 2, .. and its intended position clearly indicated in the manuscript:

\section{Figure 1 here}

The author's name in pencil must be on all separate sheets of diagrams.

A figure is expensive to reproduce and should be included only when the subject matter demands it, or when it greatly clarifies the exposition.

The Society recognizes that some authors do not have the facilities for producing drawings of a sufficiently high standard to be reproduced directly and it is therefore willing to have such diagrams redrawn, provided that they are clear.

\section{Tables}

Tables should be numbered (above the table) and set out on separate sheets. Indicate the position of each in the text as for figures:

Table 3 here

\section{References}

References should be collected at the end of the paper numbered in alphabetical order of the authors' names. Titles of journals should be abbreviated as in Mathematical Reviews. The following examples show the preferred style for references to a paper in a journal, a paper in a proceedings volume, a book and an unpublished dissertation:

[1] J. F. ADAMs. On the non-existence of elements of Hopf invariant one. Ann. of Math. (2) 72 (1960), 20-104.

[2] M. P. Fourman and D. S. ScotT. Sheaves and logic. In Applications of Sheaves, Lecture Notes in Math. vol. 753 (Springer-Verlag, 1979), pp. 302-401.

[3] P. T. Johnstone. Stone Spaces. Cambridge Studies in Advanced Math. no. 3 (Cambridge University Press, 1982).

[4] F. W. Lawvere. Functorial semantics of algebraic theories. Ph.D. thesis. Columbia University (1963). 


\section{Mathematical Proceedings of the Cambridge Philosophical Society \\ MPCPCO 119 (Pt 4) 575-762 (1996) 0305-0041 May 1996}

\section{CONTENTS}

Green Berg, P. Marked homeomorphisms and the realization problem . . . . . . 575

RYBA. A. J. E. A natural invariant algebra for the Harada-Norton group . . . . . . 597

Collins. D. J. \& Turner, E. C. All automorphisms of free groups with maximal rank fixed subgroups.

J. K. Betweenness relations and eycle-free partial orders .

Kwasik, S. \& SchULTz, R. Fundamental groups of 4-manifolds with circle actions _. . . 645

FARHADI, H-R. Isomorphisms between the second duals of group algebras of locally compact groups

RIPPON, P. J. Towards a higher-dimensional MacLane class .

Brown, G. Koumandos, S. \& W ANo, K-Y. Positivity of more Jacobi polynomial sums

FAlCon ER. K. J. \& MatTila, P. The packing dimension of projections and sections of measures .695

HowroY D, J. D. On Hausdorff and packing dimension of product spaces . . . . . $\quad$. 715

WERN ER. W. Beurling's projection theorem via one-dimensional Brownian motion $\quad . \quad 729$

REIN, G. Cosmological solutions of the Vlasov-Einstein system with spherical, plane, and hyperbolic symmetry 